\title{
Tendencia en la mortalidad por fiebre reumática aguda y cardiopatía reumática crónica en Venezuela, 1955-1994
}

\author{
Mortality trends for acute rheumatic fever and \\ chronic rheumatic heart disease in Venezuela, \\ 1955-1994
}

César I saacura 1

Ricardo Granero 1

1 Unidad de Investigación, Centro Cardiovascular Centro-Occidental - ASCARDIO. Apartado Postal 495. Barquisimeto 3002, Venezuela. jgranero@conicit.ve
A bstract This study reports on deaths dueto acuterheumatic fever (ARF) and chronic rheumatic heart di sease (CRHD) in Venezuela from 1955 to 1994. Census publications and other official sources provide the population data. Yearly age and sex-specific mortal ity rates per 100,000 inhabitants were cal culated and adjusted by the di rect method to the WHO's standard population. The RF-adjusted mortality rate (AMR) declined: total from 7.68 to 1.08 (-85.9\%); male from 7.53 to $0.84(-88.8 \%)$; and female from 7.83 to $1.33(-83.0 \%)$. In all cases female RF-AM Rs were higher than male. There was a reduction of $88.5 \%$ in the ratio of RF deaths to cardiovascular deaths (ICD-6 330-334,400-447; ICD-7 330-334,400-447; ICD-8 390-438; ICD-9 390-438). ARF-AMR declined (-93.5\%) as did CRHD-AMR (-85.1\%). Proportional death by ARF and CRHD in patients 45 years old and younger increased. Venezuela experienced a continuous declinein RF-AMR from 1955 to the 1980s, followed by a period of stabilization lasting until 1994. The reduction could be the consequence of improvement in socioeconomic status and of RF prevention with penicillin, a standard practice in the health care system. The 1980s and 1990s RF-AMR stabilization could be associated with economic setbacks in Venezuela and changes in the agent's virulence patterns. Key words Mortality; Rheumatic Fever; Rheumatic Heart Disease; Epi demi ology

Resumen Este estudio incluye defunciones por fiebre reumática aguda (FRA) y cardiopatía reumática crónica (CRC) registradas en Venezuela de 1955 a 1994, según edad y sexo. Se calcularon tasas de mortalidad por 100.000 habitantes, ajustadas según método directo a la población mundial estándar de la OMS. Se produjo un descenso en la tasa ajustada de mortalidad (TAM) por FR para ambos sexos de 7,68 a 1,08 (Variación Porcentual [VP] -85,9\%); sexo masculino de 7,53 a 0,84 (VP -88,8\%) y sexo femenino de 7,83 a 1,33 (VP -83,0\%). Las TAM fueron mayores en el sexo femenino. La proporción de muertes por FR sobre las producidas por enfermedades circuIatorias (CIE-6 330-334,400-447; CIE- 7 330-334,400-447; CIE-8 390-438; CIE-9 390-438) descendió en $88,5 \%$. Se observó, para ambos sexos, un descenso en las TAM por FRA (VP -93,5\%) y CRC (VP -85,1\%). El porcentaje de defunciones por FRA y CRC ocurridas en menores de 45 años mostró una tendencia ascendente. La reducción en Ias TAM por FR puede ser atribuiblea mejoras socioeconómicas, así como a prevención con penicilina. La estabilización en las TAM a partir de 1980 podría explicarse por el deterioro soci oeconómico experimentado en Venezuela y por la aparición de cepas más virulentas del EBHA.

Palabras clave Mortalidad; Fiebre Reumática; Cardiopatía Reumática; Epidemiología 


\section{Introducción}

Las evidencias epidemiológica, clínica e inmunológica indican que algunos tipos de estreptococo beta-hemolítico del grupo A de Lancefield (EBHA) tienen la facultad de producir fiebre reumática (FR), como complicación no supurativa de infecciones en la faringe (Muñoz, 1977). Las cepas de EBHA que causan epidemias de faringitis estreptocócica tienen mayor probabilidad de ser reumatogénicas (Stollerman, 1991). Típicamente, la fiebre reumática aguda (FRA) ocurre de 1 a 3 semanas después de la faringitis por EBHA (Muñoz, 1977).

A nivel mundial se calcula una incidencia anual de 15 a 20 millones de casos de FR, principalmente en escolares ( 5 a 15 años de edad) de sectores de bajos ingresos de naciones en desarrollo. En estas poblaciones la cardiopatía reumática constituye la principal causa de muerte por enfermedad cardíaca entre los 5 y 24 años de edad (Muñoz, 1977; Stollerman, 1991; Stollerman, 1991b; Fyler, 1994; PAHO, 1985).

La tasa de ataque de la FR después de faringitis estreptocócica sintomática es de aproximadamente $0,3 \%$ bajo condiciones endémicas y de $3 \%$ bajo condiciones epidémicas (Muñoz, 1977; Stollerman, 1991; Stollerman, 1991b; Fyler, 1994; PAHO, 1985; OMS, 1988; Muñoz, 1995). Un episodio de FR previo aumenta la tasa de ataque luego de infección estreptocócica, describiéndose hasta $50 \%$ durante el año siguiente al ataque inicial. La tasa de ataque disminuye progresivamente en los 4 o 5 años siguientes al primer ataque, estabilizándose en aproximadamente $10 \%$ por año en los subsiguientes. Se estima que un tercio de los pacientes con FRA desarrollará lesiones valvulares. No obstante, sólo la mitad de los casos de cardiopatía reumática crónica (CRC) tienen historia previa de FRA (PAHO, 1985). La frecuencia de recurrencias reumáticas luego de infecciones estreptocócicas es consistentemente mayor en pacientes con cardiopatía reumática que en aquellos sin lesiones cardíacas (Stollerman, 1991; Stollerman, 1991b).

La incidencia de FR en los países industrializados comenzó a disminuir a comienzos del siglo XX y se encuentra actualmente por debajo de 5 casos por 100.000 habitantes al año (OMS, 1988). La FRA está lejos de ser una entidad superada, como lo evidencian los brotes epidémicos esporádicos en poblaciones industrializadas, donde el entorno social y económico es satisfactorio (Ayoub, 1992). En el presente trabajo presentamos la tendencia de la mortalidad por FRA y CRC en Venezuela.

\section{Materiales y métodos}

Se incluyen las defunciones por FRA y CRC según la Clasificación Internacional de Enfermedades ( $\mathrm{CIE})$ en sus 6ạ, 7ạ, 8ạ y $9 a \underline{a}$ revisiones (CIE-6 400-402,410-416; CIE-7 400-402,410416; CIE-8 390-392,393-398; CIE-9 390-392,393398). Las defunciones por FR (sumatoria de FRA y CRC), según grupos de edad y sexo, para Venezuela, entre 1955 y 1994, se obtuvieron del Registro Central de Casos de la División de Enfermedades Cardiovasculares del Ministerio de Sanidad y Asistencia Social (MSAS). Las defunciones específicas por FRA y CRC se obtuvieron de los Anuarios de Epidemiología y Estadística Vital del MSAS. Debido a la dificultad para obtener todos los anuarios de la serie cronológica 1955-1994, se decidió presentar las tasas correspondientes a los años 1955, 1966, 1975, 1985 y 1994, los cuales son aproximadamente equidistantes (MSAS, 1994).

Las tasas crudas de mortalidad específicas por sexo fueron calculadas empleando estimaciones de población basadas en los censos oficiales de población y vivienda (años 1950, 1961, 1971, 1981 y 1990) (OCEI, 1990). También se utilizaron datos proporcionados por la División de Epidemiología del MSAS y por la Fundación para el Desarrollo de la Región CentroOccidental de Venezuela (FUDECO). Las estimaciones de población intercensales fueron calculadas por interpolación lineal.

Las tasas de mortalidad por FR, según sexo, por 100.000 habitantes fueron ajustadas según el método directo a la población mundial estándar de la Organización Mundial de la Salud (Colton, 1974). Se calculó la variación porcentual (VP) en las tasas ajustadas de mortalidad (TAM) entre el primer año y el último año de cada serie estudiada.

Las TAM fueron graficadas siguiendo el método de promedios móviles de 3 años. Este método produce una curva con un punto por cada año. El primer punto es el promedio de las TAM de los dos primeros años de la serie. El último punto del gráfico es el promedio de las TAM de los dos últimos años de la serie. Los otros puntos del gráfico corresponden al promedio de las TAM de tres años: la del año que coincide con el punto, la del año inmediato anterior y la del año inmediato posterior (Folsom et al., 1987).

La magnitud de la mortalidad por FR relativa a la mortalidad cardiovascular se presenta como el porcentaje de las defunciones por FR sobre las producidas por afecciones circulatorias (enfermedades cardíacas, hipertensivas y cerebrovasculares: CIE-6 330-334,400-447; CIE- 
7 330-334,400-447; CIE-8 390-438; CIE-9 390438). Se determinó el porcentaje de las defunciones por FRA y CRC que ocurrieron en menores de 45 años.

\section{Resultados}

En el período 1955-1994, se produjo un descenso en la TAM por FR en Venezuela, para ambos sexos, de 7,68 a 1,08 (VP -85,9\%); sexo masculino de 7,53 a 0,84 (VP - 88,8\%) y sexo femenino, de 7,83 a 1,33 (VP -83,0\%). La figura 1 muestra la tendencia de laTAM por FR (promedios móviles de 3 años). Se observan un descenso sostenido hasta el final de la década de los setenta y una estabilización a partir de 1980. Las TAM correspondientes al sexo femenino son consistentemente mayores que las correspondientes al sexo masculino, en todo el período estudiado. En 1955, la FR fue responsable del $5,2 \%$ del total de muertes por afecciones circulatorias, mientras que, en 1994, esa cifra desciende a $0,6 \%$, una VP de $-88,5 \%$. El descenso en la TAM por FR en Venezuela ha sido, en promedio, 0,17 por 100.000 habitantes, por año.

Las tablas 1 y 2 muestran un descenso en las TAM por FRA (VP -93,5\%) y CRC (VP -85,1\%) en el período 1955-1994. Esta tendencia se mantiene al considerar las TAM específicas por sexo. Las TAM por CRC son mayores en el sexo femenino, mientras que, en las de FRA, no existe predominio franco de ningún sexo. El porcentaje de las defunciones por FRA que ocurrieron en menores de 45 años es mayor al correspondiente por CRC, apreciándose, en ambos casos, una tendencia ascendente de este indicador.

\section{Discusión}

Para los clínicos de países desarrollados, es difícil encontrar un caso de FR en su vida profesional. En los Estados Unidos de América (EUA), Canadá y España, la incidencia y prevalencia de FR ha descendido abruptamente, desde la década de 1960 hasta la de 1980 (Stollerman, 1991b; Rabkin \& Chu-Chun-Lin, 1988; Cortina et al., 1991). La reducción en la mortalidad por FR también se ha producido en países menos desarrollados y aún en países subdesarrollados. De 1955 a 1973, Chile experimentó $22,8 \%$ de reducción en la tasa de mortalidad por FR por 100.000 habitantes (Muñoz, 1977). De 1963 a la década de los ochenta, Cuba experimentó una reducción en la tasa de mortalidad por FR $(6,0$ a 2,0), con una tasa por FRA menor de 0,2 por 100.000 habitantes (Nordet et al., 1991). Similarmente, el descenso en la TAM por FR en Venezuela ha sido, en promedio, 0,17 por 100.000 habitantes, por año.

Se han propuesto diferentes explicaciones para el descenso en la incidencia por FR. Entre ellas podemos citar la mejoría de las condiciones sociales y económicas, el tratamiento preventivo de la FR con antibióticos y cambios en la distribución geográfica y virulencia de serotipos del EBHA (Stollerman, 1991b; PAHO, 1985; Kassem \& Zaher, 1992). En los últimos

Figura 1

Fiebre reumática: promedios móviles de tasas de mortalidad ajustadas a la población mundial estándar. Venezuela, 1955-1994

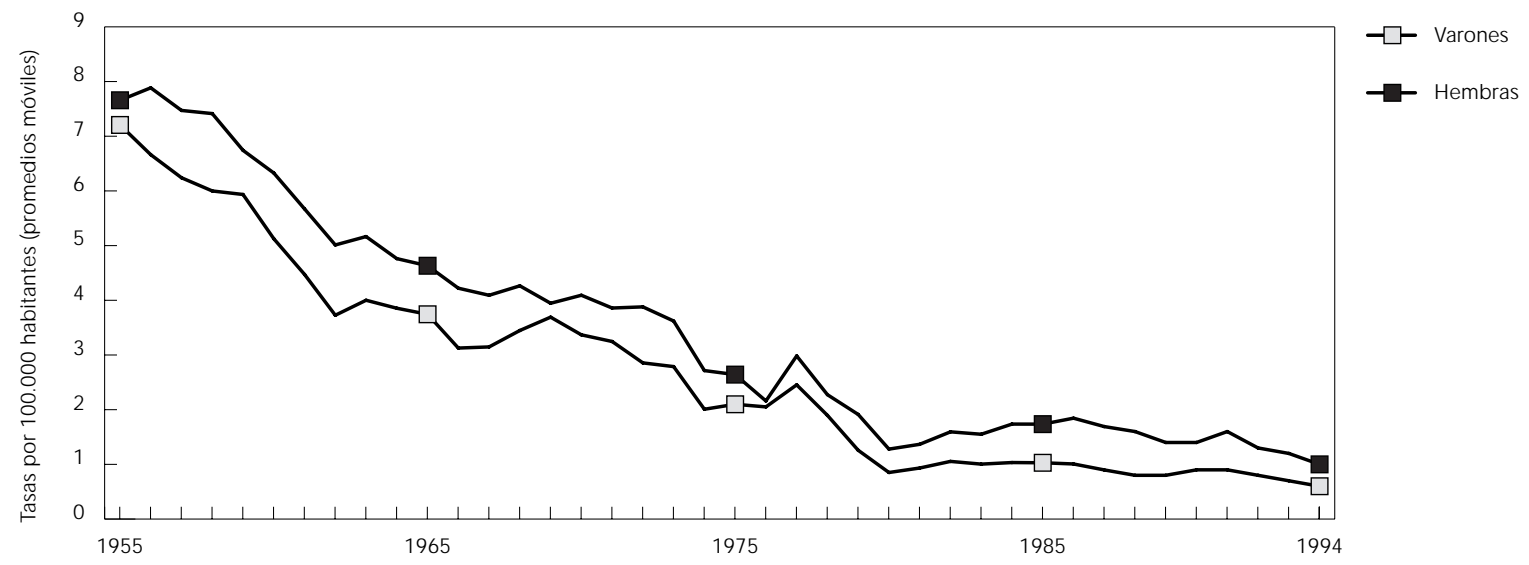


Tabla 1

Fiebre reumática aguda: defunciones según sexo y tasas estandarizadas de mortalidad por 100.000 habitantes. Venezuela: 1955, 1966, 1975, 1985 y 1994.

\begin{tabular}{|c|c|c|c|c|c|c|c|}
\hline \multirow[t]{2}{*}{ Año } & \multicolumn{2}{|c|}{ Varones } & \multicolumn{2}{|c|}{ Hembras } & \multicolumn{2}{|c|}{ Ambos Sexos } & \multirow[t]{2}{*}{$\%<45$ años } \\
\hline & Muertes & Tasa & Muertes & Tasa & Muertes & Tasa & \\
\hline 1955 & 16 & 0.70 & 14 & 0.53 & 30 & 0.62 & 56.7 \\
\hline 1966 & 8 & 0.16 & 19 & 0.53 & 27 & 0.35 & 77.8 \\
\hline 1975 & 84 & 0.22 & 119 & 0.28 & 203 & 0.25 & 80.0 \\
\hline 1985 & 8 & 0.08 & 5 & 0.05 & 13 & 0.06 & 100.0 \\
\hline 1994 & 4 & 0.04 & 3 & 0.03 & 7 & 0.04 & 71.4 \\
\hline
\end{tabular}

$\%<45$ años = porcentaje de las defunciones por FRA que ocurrieron en menores de 45 años. Ajuste según la población mundial estándar de la O rganización Mundial de la Salud.

Fuente: Anuarios de Epidemiología y Estadística Vital, M inisterio de Sanidad.

Tabla 2

Cardiopatía reumática crónica: defunciones según sexo y tasas estandarizadas de mortalidad por 100.000 habitantes. Venezuela: 1955, 1966, 1975, 1985 y 1994.

\begin{tabular}{cccccccc}
\hline \multirow{2}{*}{ Año } & \multicolumn{2}{c}{ Varones } & \multicolumn{2}{c}{ Hembras } & \multicolumn{2}{c}{ Ambos Sexos } & \multirow{2}{*}{ \% } \\
& Muertes & Tasa & Muertes & Tasa & Muertes & Tasa \\
& 113 & 6.83 & 123 & 7.30 & 236 & 7.06 & 26.3 \\
1955 & 76 & 2.65 & 100 & 3.42 & 176 & 3.04 & 36.4 \\
1966 & 203 & 0.78 & 47 & 0.78 & 250 & 0.78 & 44.5 \\
1975 & 82 & 1.22 & 138 & 2.10 & 220 & 1.66 & 45.5 \\
1985 & 71 & 0.80 & 110 & 1.30 & 181 & 1.05 & 47.0 \\
1994 & & & & & & & \\
\hline
\end{tabular}

$\%<45$ años = porcentaje de las defunciones por Cardiopatía Reumática Crónica que ocurrieron en menores de 45 años. Ajuste según la población mundial estándar de la Organización Mundial de la Salud.

Fuente: Anuarios de Epidemiología y Estadística Vital, Ministerio de Sanidad.

150 años, se ha observado una tendencia a la reducción de la $\mathrm{FR}$, precediendo por muchas décadas el uso de la penicilina como medida preventiva, lo que resalta el papel ejercido por los cambios socioeconómicos (Quinn, 1989), tendencia que ha sido fortalecida por el uso de antibióticos en la prevención de FR. Por ejemplo, Costa Rica experimentó $85 \%$ de reducción en la incidencia de FR entre 1970 y 1980, luego de introducir la penicilina benzatínica en la prevención primaria de FR (Moha, 1985; Mclaren et al., 1994). De la misma manera, en Boston (EUA), se evidenció que la mortalidad debida a carditis reumática descendió rápidamente a cero desde el advenimiento de la penicilina, alrededor de 1946 (Massell et al., 1988).

En Venezuela, la reducción en las TAM por FR, desde 1955 hasta la década de los ochenta, puede ser atribuible a mejoras socioeconómicas, así como a la prevención primaria y secundaria con penicilina, comúnmente aplicada por el sistema de salud, y al avance tecnológico en el tratamiento de las complicaciones de FR. La estabilización en las TAM a partir de 1980 podría explicarse en virtud del deterioro de la condición socioeconómica del país en este período y de la aparición de cepas más virulentas del EBHA, las cuales han sido detectadas en otros países (Cleary et al., 1992). De igual forma, se ha observado, en otros países que las tasas de mortalidad por FR del sexo femenino son consistentemente mayores que las del sexo masculino (Rabkin \& Chu-Chun-Lin, 1988).

En nuestros resultados se observa una tendencia ascendente en el porcentaje de las defunciones por FRA y CRC que ocurrieron en menores de 45 años. Esto podría ser consecuencia de dos hechos: a) la aparición de cepas más virulentas que condicionarían una mayor letalidad en este grupo etario; b) una disminución en la mortalidad por CRC en mayores de 45 años, debido a la prevención secundaria y a los avances tecnológicos en el tratamiento de las secuelas. 
Si bien la FR ha descendido notablemente, se mantiene la posibilidad de una resurgencia de la enfermedad, como lo demuestran los brotes esporádicos detectados en países industrializados (Ayoub, 1992). Cabe mantener una vigilancia epidemiológica constante, en razón del deterioro socioeconómico que experimenta Venezuela, lo cual podría promover un repunte de esta enfermedad.

\section{Referencias}

AYOUB, E. M., 1992. Resurgence of rheumatic fever in the United States. The changing picture of a preventable illness. Postgraduate Medicine, 92:133136.

CLEARY, P. P.; KAPLAN, E. L.; HANDLEY, J. P.; WLAZLO, A.; KIM, M. H.; HAUSER, A. R. \& SCHLIEVERT, P. M., 1992. Clonal basis for resurgence of serious Streptococcus pyogenes disease in the 1980s. The Lancet, 339:518-521.

COLTON, T., 1974. Statistics in Medicine. Boston: Little, Brown and Company.

CORTINA GREUS, P.; ALFONSO SANCHEZ, J. L.; CORTES VIZCAINO, C.; SMEYERS DURA, P. \& GONZALEZ ARRAEZ, J. I., 1991. Epidemiological course of rheumatic fever and rheumatic heart disease in Spain (1951-1986). Revista de Sanidad eHigiene Pública de Madrid, 65:17-24.

FOLSOM. A.; GOMEZ-MARIN, O.; GILLUM, R.; LOHMAN, W. \& JACOBS, D., 1987. Out-of-hospital coronary death in an urban population-validation of death certificate diagnosis. American Journal of Epidemiology, 125:1012-1018.

FYLER, D. C., 1994. Fiebre reumática. In: NADAS Cardiología Pediátrica (D. C. Fyler, ed.), pp. 305-318, Madrid: Mosby, División de Times Mirror de España, S.A.

KASSEM, A. S. \& ZAHER, S. R., 1992. An international comparison of the prevalence of streptococcal infections and rheumatic fever in children. Pediatric Annals, 21:835-842.

MASSELL, B. F.; CHUTE, C. G.; WALKER, A. M. \& KURLAND, G. S., 1988. Penicillin and the marked decrease in morbidity and mortality from rheumatic fever in the United States. New England Journal of Medicine, 318:280-286.

MCLAREN, M. J.; MARKOWITZ, M. \& GERBER, M., 1994. Rheumatic heart disease in developing countries: the consequence of inadequate prevention. Annals of Internal Medicine, 120:243245.

MSAS (Ministerio de Sanidad y Asistencia Social), 1994. Anuarios de Epidemi ología y Estadística Vital Años 1955, 1966, 1975, 1985, 1994. Caracas: MSAS.

MOHA, E., 1985 La fiebre reumática en Costa Rica. Revista Médica del Hospital Nacional de Niños (Costa Rica), 20:77-86.
MUÑOZ ARMAS, S., 1977. Fiebre Reumática y Enfermedad Reumática del Corazón. Caracas: Fondo Editorial Común S.C.

MUÑOZ OBANDO, H, 1995. Fiebre Reumática. In: Manual de Cardiología Clínica (D. Almeida Feo \& S. Brandi Pifano, ed.), Tomo 2, pp. 213-238, Caracas: Consejo de Desarrollo Científico y Humanístico de la Universidad Central de Venezuela/Fondo Andrés Bello.

NORDET CARDONA, P.; LOPEZ, R.; LA LLAVE, G.; MUÑOS, R.; CASTRO, E. \& ROJAS, J., 1991. Fiebre reumática: incidencia, prevalencia y aspectos clínicoepidemiológicos. Revista Cubana de Cardiología y Cirugía Cardiovascular, 5:25-33.

OCEI (Oficina Central de Estadística e Informática), 1990. Censo General de Población y Vivienda de Venezuela Años 1950, 1961, 1971, 1981 y 1990. Caracas: OCEI.

OMS (Organización Mundial de la Salud), 1988. Fie bre Reumática y Cardiopatía Reumática. Informe de un Grupo de Estudio dela OMS. Ginebra: OMS, Serie de Informes Técnicos 764.

PAHO (Pan American Health Organization), 1985. Prevention and Control of Rheumatic Fever in the Community. Manual of Operational Standards for a Program to Extend Coverage at Different Levels of Care. Washington: PAHO, Scientific Publication ㄲo 399.

QUINN, R. W., 1989. Comprehensive review of morbidity and mortality trends for rheumatic fever, streptococcal disease, and scarlet fever: the decline of rheumatic fever. Review of Infectious Disease, 11:928-953.

RABKIN, S. W. \& CHU-CHUN-LIN, S.F., 1988. Epidemiology of valvular heart disease in Canada. Canadian Journal of Cardiology, 4:412-416.

STOLLERM AN, G. H., 1991. Rheumatic Fever. In: Harrison's Principles of Internal Medicine (J. D. Wilson, E. Braunwald, K. J. Isselbacher, R. G. Petersdorf, J. B. Martin, A. S. Fauci \& R. K. Root, ed.), Volume 1, pp. 933-8, New York/London: McGraw Hill, Inc.

STOLLERM AN, G.H., 1991. Rheumatic Fever and Other Rheumatic Diseases of the Heart. In: Heart Disease. A Textbook of Cardiovascular Medicine (E. Braunwald, ed.), Volume 2, pp. 1721-41, Philadelphia: W.B. Saunders Company. 